\title{
SHELL EFFECTS AND THE FLUID DYNAMICAL MODEL FOR NUCLEAR GIANT RESONANCES
}

\author{
M. BRACK \\ Institute of Theoretical Physics, University of Regensburg, D.8400 Regensburg, Fed. Rep. Germany
}

Received 2 January 1983

\begin{abstract}
We discuss the fluid dynamical approach to nuclear giant resonances using imposed scaling type oscillations. We calculate the response of the intrinsic kinetic energy to the isoscalar twist mode $\left(J^{\pi}=2^{-}\right)$quantum mechanically and show that for this mode as well as for the lowest normal parity modes $\left(J^{\pi}=0^{+}, 1^{-}\right.$and $\left.2^{+}\right)$, the static single particle effects lead to the same restoring forces as they have been obtained in semiclassical calculations from the distortions of the local Fermi surface in momentum space
\end{abstract}

The refined experimental measurements of nuclear giant resonances (see ref. [1] for a recent review) have raised much interest in the theoretical description of these excitation modes within nuclear fluid dynamical models [1-4]. Studying imposed collective oscillations of the nucleus defined through generalized scaling transformations [5] of the wave functions, Holzwarth and Eckart [3] (in the following abbreviated as HE) stressed the importance of what they called dynamical corrections to the ThomasFermi method due to distortions of the local Fermi surface in momentum space. These lead to additional restoring forces, compared to usual hydrodynamics, for normal parity modes with $J \geqslant 2$ and for all anomal parity modes. The close connection of this mechanism to that of zero sound was stressed by Eckart et al. [4] and further discussed in detail by Jennings and Jackson [6].

In this note we shall focus on the twist mode $\left(J^{\pi}=\right.$ $2^{-}$) and demonstrate that for this mode, as well as for the few lowest normal parity modes, the additional restoring forces derived by $\mathrm{HE}$ from the distortion of the Fermi surface are identical with those obtained if the static single particle or shell effects of the intrinsic kunetic energy are taken into account in an exact quantum-mechanical way. For notational simplicity, we shall use the independent particle (Hartree-Fock) picture; the arguments are unchanged if one starts from many-body wave functions as done in ref. [3].

The essential assumption of the imposed collective scaling modes is that during the oscillations of the nucleus, all static wave functions are scaled in phase according to

$\varphi_{l}(r) \rightarrow \varphi_{l}(\alpha, r)=\exp \left[\alpha\left(\frac{1}{2} \nabla \cdot u+u \cdot \nabla\right)\right] \varphi_{i}(r)$,

so that the only true dependence of the dynamical wave functions $\varphi_{i}(\alpha, r)$ comes through the collective variable $\alpha=\alpha(t)$. Hereby $\boldsymbol{u}=\boldsymbol{u}(\boldsymbol{r})$ is the velocity field in units of $\dot{\alpha}$, corresponding to the usual small amplitude assumption $v(r, t)=\dot{\alpha}(t) u(r)$. The dynamical local density $\rho$ and kinetic energy density $\tau$ are defined as usually by

$\rho(\alpha, r)=\sum_{i=1}^{A}\left|\varphi_{i}(\alpha, r)\right|^{2}$,

$\tau(\alpha, r)=\left(h^{2} / 2 m\right) \sum_{l=1}^{A}\left|\nabla_{r} \varphi_{l}(\alpha, r)\right|^{2}$,

and the total intrinsic kinetic energy is then given by

$T_{\alpha}=\int \tau(\alpha, r) \mathrm{d}^{3} r$.

HE [3] calculated $T_{\alpha}$ semiclassically using the extended Thomas-Fermi approximation [7] of the Wigner distribution function, hereby taking explicitly into account the deformation of the momentum distribution. However, there is a prior1 no need for this semiclassical treatment, since the simplicity of the scaling transformations eq. (1) for the modes with 
lowest multiplicity allows to express the scaled wave functions $\varphi_{l}(\alpha, r)$ in a closed form as the static $\varphi_{i}\left(r^{\prime}\right)$ as functions of transformed coordinates $r^{\prime}(\alpha)$ (times a multiplicative factor in the monopole case).

We shall restrict ourselves to isoscalar modes only and list in the following, for the sake of completeness also for normal parity modes with $J^{\pi}=0^{+}, 1^{-}$and $2^{+}$, the velocity fields $u(r)$ together with the scaled wave functions $\varphi_{i}(\alpha, r)$. (We use the same symbol for the collective variable $\alpha$ in all modes; the fact that it has different dimensions in different cases should not cause confusion.)

Monopole

$u=r=(x, y, z), \varphi_{l}(\alpha, r)=\mathrm{e}^{3 \alpha / 2} \varphi_{i}\left(\mathrm{e}^{\alpha} x, \mathrm{e}^{\alpha} y, \mathrm{e}^{\alpha} z\right) ;$

dipole:

$$
u=z=(0,0,1), \varphi_{i}(\alpha, r)=\varphi_{i}(x, y, z+\alpha) ;
$$

axial quadrupole:

$$
u=(x, y,-2 z), \quad \varphi_{i}(\alpha, r)=\varphi_{i}\left(\mathrm{e}^{\alpha} x, \mathrm{e}^{\alpha} y, \mathrm{e}^{-2 \alpha} z\right) ;
$$

nonaxıal quadrupole:

$$
u=(x,-y, 0), \quad \varphi_{i}(\alpha, r)=\varphi_{i}\left(\mathrm{e}^{\alpha} x, \mathrm{e}^{-\alpha} y, z\right) ;
$$

twist:

$$
u=(z y,-z x, 0), \varphi_{i}(\alpha, r)=\varphi_{i}\left(x^{\prime}, y^{\prime}, z^{\prime}\right) .
$$

(Note that these results are exact to all powers in $\alpha$.) For the twist mode, the coordinates $r^{\prime}$ are the result, of a rotation around the $z$ axis about an angle $\omega=\alpha z$ :

$x^{\prime}=x \cos (\alpha z)+y \sin (\alpha z)$,

$y^{\prime}=-x \sin (\alpha z)+y \cos (\alpha z)$,

$z^{\prime}=z$.

Insertıng the explic1t forms of $\varphi_{i}(\alpha, r)$ of eq. (4) into $\tau(\alpha, r)$ eq. (2), it is a straightforward matter to calculate the total kinetic energy $T_{\alpha}$, eq. (3) as an integral over derivatives of the unscaled functions $\varphi_{i}(r)$ and some explicit functions of $\alpha$. If one assumes the nucleus in its equilibrium state at $\alpha=0$ to be spherical with filled $j$ shells, one gets for the normal parity modes the following results for the exact kinetic energy $T_{\alpha}$ :

$T_{\alpha}=T_{0} f(\alpha)$,

with

$$
\begin{array}{ll}
\text { monopole } & f(\alpha)=\mathrm{e}^{2 \alpha} \\
\text { dipole: } & f(\alpha)=1
\end{array}
$$

axial quadrupole: $\quad f(\alpha)=\frac{1}{3}\left(2 \mathrm{e}^{2 \alpha}+\mathrm{e}^{-4 \alpha}\right)$ nonaxial quadrupole: $f(\alpha)=\frac{1}{3}\left(1+\mathrm{e}^{2 \alpha}+\mathrm{e}^{-2 \alpha}\right)$.

For the monopole, the dipole and the axial quadrupole, these results were also obtained in connection with the sum rule approach [8]. Exactly the same relations between the semiclassical kinetic energies $T_{\alpha}$ and $T_{0}$ were obtained by HE [3].

For the twist mode, we find with eqs. (4) and (5)

$$
\begin{aligned}
& \left|\nabla_{r} \varphi_{i}(\alpha, r)\right|^{2}=\left|\nabla_{r^{\prime}} \varphi_{l}\left(r^{\prime}\right)\right|^{2} \\
& \left.\quad+2 \alpha \hbar^{-1} \operatorname{Im}\left\{\partial \varphi_{l}^{*}\left(x^{\prime}\right) / \partial z^{\prime}\right] \hat{l}_{z^{\prime}} \varphi_{l}\left(r^{\prime}\right)\right\} \\
& \quad+\alpha^{2} \hbar^{-2}\left|\hat{l}_{z^{\prime}} \varphi_{l}\left(r^{\prime}\right)\right|^{2},
\end{aligned}
$$

which is again exact to all orders in $\alpha$. If the system has axial symmetry around the $z$ axis, the term linear in $\alpha$ in eq. (8) is identically zero, and we obtain for the exact kinetic energy

$T_{\alpha}=T_{0}+\left(\alpha^{2} / 2 m\right) \sum_{i=1}^{A}\left\langle i\left|\hat{l}_{z}^{2}\right| i\right\rangle$.

For a spherical nucleus with filled $J$-shells, we get

$T_{\alpha}=T_{0}+\alpha^{2}\left(\hbar^{2} / 2 m\right) \frac{1}{3} \sum_{j}(2 \jmath+1) l_{j}\left(l_{j}+1\right)$,

where the sum goes over the occupied $j$-shells and $l$ is the orbital angular momentum belonging to each shell. (Note that the s-states do not contribute to the restoring force in $T_{\alpha}$ !)

We now proceed to calculate the sum of matrix elements in eq. (9) semiclassically by writing

$\sum_{i=1}^{A}\left\langle\imath\left|\hat{l}_{z}^{2}\right| i\right\rangle=\int \mathrm{d}^{3} r\left[\hat{l}_{z} \hat{l}_{z}^{+} \cdot p\left(r, r^{\prime}\right)\right]_{r=r^{\prime}}$,

$\rho\left(r, r^{\prime}\right)=\sum_{i=1}^{A} \varphi_{l}^{*}\left(r^{\prime}\right) \varphi_{l}(r)$

and replacing the density matrux $\rho\left(r, r^{\prime}\right)$ by its wellknown Thomas-Fermi expression:

$\rho_{\mathrm{TF}}\left(r, r^{\prime}\right)=\rho_{\mathrm{TF}}(R)\left(3 / s k_{\mathrm{F}}\right) j_{1}\left(s k_{\mathrm{F}}\right)$,

where $\rho_{\mathrm{TF}}=2 k_{\mathrm{F}}^{3} / 3 \pi^{2}, k_{\mathrm{F}}=2 m\left(\lambda-V(R) / \hbar^{2}\right]^{1 / 2}$ is the local Fermi momentum, $V(r)$ is the one-body 
(Hartree-Fock) potential and $R=\frac{1}{2}\left(r+r^{\prime}\right), s=\left|r-r^{\prime}\right|$. Keeping only the lowest order term in $\hbar$, we obtain

$\left.\frac{1}{2} m^{-1} l_{z} l_{z^{\prime}}^{+} \rho_{\mathrm{TF}}\left(r, r^{\prime}\right)\right|_{r=r^{\prime}}=\frac{2}{15} \pi^{-2} k_{\mathrm{F}}^{5}\left(x^{2}+y^{2}\right) \hbar^{2} / 2 m$

$$
=\frac{1}{3}\left(x^{2}+y^{2}\right) \tau_{\mathrm{TF}}(r),
$$

and thus

$T_{\alpha}^{\mathrm{TF}}=T_{0}^{\mathrm{TF}}+\alpha^{2} \frac{1}{3} \int \mathrm{d}^{3} r\left(x^{2}+y^{2}\right) \tau_{\mathrm{TF}}(r)$,

which is the result of HE [3]. We have thus shown for the twist mode, too, that the approach of HE using the distortion of the Fermi surface is no more and no less than a clever way of taking into account the shell effects in the intrinsic kınetic energy (which are just the static ones!), without having to deal with wave functions.

The giant resonance (peak) energies are identified in the fludd dynamical scaling model with the oscillator frequencies

$E_{\mathrm{GR}}=\hbar \omega=\hbar(C / B)^{1 / 2}$.

The mass parameters are usually calculated according to the hydrodynamical expression

$B=m \int \mathrm{d}^{3} r u^{2}(r) \rho(r)$.

However, as shown by Abgrall et al. [9] for all the normal parity scaling modes discussed above, ident1cally the same mass parameters are also obtained with the purely microscopical Inglis-cranking formula [if the quantum-mechanical densities $\rho$ are used in eq (17)]. For the quadrupole and twist modes, the main contribution to the "spring constants" $C$, defined by

$C=\left.\left(\partial^{2} / \partial \alpha^{2}\right) \int \mathrm{d}^{3} r \mathcal{E}_{\mathrm{intr}}(\alpha, r)\right|_{\alpha=0}$,

comes from the intrinsic kinetic energy $T_{\alpha}$. Surface and $\tau$-dependent terms in the potential energy as well as the spin--orbit energy give relatively small corrections (the latter two also for the twist mode!). The main success of the fluid dynamical approach with the imposed scaling modes lies in the fact that the correct factor $f(\alpha)$ in eq. (6) - which is different in usual hydrodynamics - is necessary to obtain the correct order of magnitude and the approximate $A^{-1 / 3} \mathrm{de}$ pendence of the giant quadrupole resonance energy.

In a self-consistent fluid dynamical description [4] the collective modes are not imposed but determined by solving the dynamical Euler variational equations. The numerical results of $\mathrm{HE}$ and collaborators [4] show, the variational eigenfrequencies $\hbar \omega\left(J^{\pi}\right)$ being only some $5-10 \%$ lower than those of the imposed scaling modes, that the essential features of the giant resonance modes are rather well described by the scaling model. The main source for the restoring forces is, in the self-consistent approach, too, the distortion of the local Fermı surface. Thus, the qualitative picture is not changed in the more refined variational approach.

For the understanding of the mechanisms leading to the giant resonances in magic closed-shell nuclei, we believe it to be rather important to realize that the fluid dynamical model, in its essential input, is completely equivalent to that defined by the collective hamiltonian

$H(\alpha)=\frac{1}{2} B \dot{\alpha}^{2}+V(\alpha)$,

using for $V(\alpha)$ the scaled static intrinsic (e.g. HartreeFock) energy including shell effects, and for $B$ the corresponding cranking mass parameters, the whole quantized in the harmonic approximation. In this picture, the essential dynamical input is not the deformation of the local Fermi surface, since this is just a consequence of static shell effects, but the very assumption of the scaling like nature of the collective modes under consideration.

Our interpretation is in no way contradictory to the fluid dynamical model and its interpretation in terms of zero sound $[4,6]$. On the contrary, it allows for a unification with the standard view point of the shell-correction method [10], where the static deformation energy - i.e. here $V(\alpha)$ in eq. $(19)-1$ a sum of a smooth, liquid drop model type part fully compatible with the extended Thomas-Fermi model (see ref. [11]), and a fluctuating part coming from the single-particle structure such as it is precisely included in the above expressions of the intrinsic kinetic energy $T_{\alpha}$. The scaling model is, of course, only able to describe that part of the static deformation energy $V(\alpha)$ around the minumum in which no level crossings occur at the Fermi energy. It therefore works especially well in pronouncedly magic spherical nucle1 where the shell effect is sufficiently strong so that no level crossings occur during small-amplitude oscillations.

In large-amplitude processes, such as fission, where 
many level crossings occur and the single particle states are reoccupied - at least in the adiabatic limit restoring forces and inertial parameters are changed from their fluid dynamical values and strongly depend on residual interactions. The description of these effects may be attempted by including quasiparticle components in the fluid (or single-particle) dynamical model. Such an approach is presently under investigation and will be published elsewhere [12].

Encouraging discussions with K. Goeke, A. Góźdź, P.G. Reinhard and E. Werner are gratefully acknowledged.

\section{References}

[1] J. Speth and A van der Woude, Rep. Prog. Phys. 44 (1981) 719

[2] G. Bertsch, Nucl. Phys. A249 (1975) 253; C Y Wong, J A. Maruhn and T.A. Welton, Nucl. Phys. A253 (1975) 469,

H. Sagawa and G. Holzwarth, Progr. Theor. Phys 59 (1978) 1213.

[3] G. Holzwarth and G Eckart, Z. Phys. A283 (1977) 219; A284 (1978) 291.
[4] G. Holzwarth and G. Eckart, Nucl. Phys. A325 (1979) 1 , G Eckart et al., Phys. Lett. 94B (1980) 453, Nucl Phys A364 (1981) 1

[5] L.J Tassie, Austr J Phys 9 (1956) 407.

[6] B K. Jennings and A D Jackson, Phys. Rep. 66 (1980) 141.

[7] D.A. Kirzhnits, Field theoretical methods in many body systems (Pergamon, New York, 1967); M. Brack, B.K. Jennıngs and Y.H Chu, Phys. Lett. 65B (1976) 1

[8] D.M. Brınk and R. Leonard1, Nucl Phys A258 (1975) 285 , O Bohigas, A.M Lane and J. Martorell, Phys. Rep. 51 (1979) 267

[9] Y. Abgrall et al, Nucl Phys A346 (1980) 431

[10] V M. Strutinsky, Nucl Phys. A95 (1967) 420; A122 (1968) 1 , M. Brack et al., Rev Mod. Phys. 44 (1972) 320

[11] B.K. Jennings, R.K. Bhadurl and M Brack, Nucl. Phys. A253 (1975) 29;

M Brack and P. Quentun, Nucl. Phys. A361 (1981) 35, J Bartel et al, Nucl Phys A386 (1982) 79

[12] V.M. Strutinsky, A. Magner and M Brack, to be published. 